\title{
IS MULTIPLE TEAM MEMBERSHIP A CHALLENGE OR A HINDRANCE FOR INDIVIDUAL EMPLOYEES?
}

\author{
HENDRIK J. VAN DE BRAKE \\ University of Groningen, Department of HRM \& OB \\ Nettelbosje 2, 9747 AE Groningen, The Netherlands \\ FRANK WALTER \\ Justus-Liebig-University Giessen, Germany

\begin{abstract}
FLOOR A. RINK, PETER J.M.D. ESSENS \& GERBEN S. VAN DER VEGT
University of Groningen, the Netherlands
\end{abstract}

\section{INTRODUCTION}

One of the most prominent, yet largely unexplored, aspects of contemporary work is that many employees are involved in more than one team at the same time (O'Leary, Woolley, \& Mortensen, 2011a; Mortensen, Woolley, \& O’Leary, 2007). In project-based work, for example, parallel project teams often share the same personnel stock (Zika-Viktorsson, Sundstrom, \& Engwall, 2006). Similarly, health care professionals often work concurrently in multiple patient care teams (Wagner, 2000), and knowledge workers are sometimes involved in up to nine or more concurrent R\&D teams (Bertolotti, Mattarelli, Vignoli, \& Macrì, 2015).

There are good theoretical reasons to assume that MTM may have profound impacts on employees' wellbeing and job performance. Indeed, some scholars have pointed to MTM as a potentially positive experience that creates opportunities for networking, learning, and task mastery (Mortensen et al., 2007; Podsakoff, LePine, \& LePine, 2007). In direct contrast, other scholars have referred to MTM as a highly demanding and potentially stressful work practice that may reduce employees' overall functioning by complicating team roles, time schedules, and social environments (Leroy, 2009; Kauppila, 2014; Matthews et al., 2012). To resolve this apparent contradiction, we believe a better understanding of MTM's consequences for individual employees' well-being and job performance requires taking into account not only MTM's potentially positive or negative effects, but also to consider moderating factors that may shape individuals' differential reactions to similar levels of MTM.

Drawing from the challenge-hindrance stressor framework (LePine, LePine, \& Jackson, 2004), we argue that some individuals may appraise MTM as a positive challenge, reflected in the perceived importance of their work and the opportunity to use their abilities in their job. Other individuals, however, may appraise MTM as a hindrance, reflected in feelings of uncertainty about how work should be done and how one is evaluated. Incorporating insights from organizational socialization theory (Louis, 1980; Moreland \& Levine, 1982), we further posit that the extent to which individual employees will appraise MTM as a challenge or a hindrance will depend on their organizational tenure. This positive or negative appraisal, in turn, is argued to shape individual employees' job performance and absenteeism.

We tested our conceptual model using multisource data from a large organization in applied research in the Netherlands. By doing so, this study advances a more complete picture of the potential risks and rewards associated with MTM, and it provides organizations with key insights that may help to create more favorable working experiences for their employees. 


\section{THEORY AND HYPOTHESES}

We cast MTM as an individual-level phenomenon that occurs when an employee is involved in more than one team at the same time and that varies with the number of teams in which an employee is concurrently involved (Mortensen, Woolley, \& O'Leary, 2007). As such, MTM is formally defined as an individuals' number of simultaneous and active team memberships, reflected in the number of teams to which an individual allocates working time within a certain period (e.g., per week; O'Leary et al., 2011a).

MTM is inherently challenging because it requires employees to focus on various team contexts with different co-workers, task responsibilities, and supervisors (Pluut et al., 2015). Importantly, research suggests that employees may perceive these challenges as temporary obstacles that can be dealt with through hard work and task mastery (i.e., as something positive rather than negative; LePine, LePine, \& Jackson, 2004). Empirical work in the job design, creativity, and motivation literature also suggests that MTM may promote interesting and intrinsically rewarding experiences for employees (Amabile et al., 1996). MTM may, for example, result in the feeling that one's expertise is needed in a number of concurrent projects and therefore contributes to the success of the organization as a whole (Cummings \& Haas, 2012). Similarly, multi-teamers usually hold important roles within their respective teams, as they can function as "network brokers" (Aldrich \& Herker, 1977; Burt, 2009) that combine and integrate information from separate teams to create innovative solutions and task approaches (Grant, 1996). Taken together, it appears that multi-teamers are subject to a multitude of highly challenging, meaningful and important work experiences. Hence, we hypothesize:

Hypothesis 1. An employee's multiple team membership is positively related to his or her challenge appraisals.

At the same time, there are valid reasons why employees might perceive MTM as a hindrance. Employees appraise a situation as a hindrance when it harms their personal goal accomplishment (Lazarus \& Folkman, 1984). In organizational settings, such perceptions may result from uncertainty about the responsibilities and behaviors required to perform a work role (i.e., role ambiguity; Kauppila, 2014). Because multi-teamers need to cooperate with a relatively large number of different supervisors and teammates (O'Leary et al., 2011a), it may be more difficult for them to fully understand the expectations that go along with each of these multiple team roles. In addition, MTM curtails the time an employee can spend within any given team (O'Leary et al., 2011a). As a result, multi-teamers have fewer opportunities to get acquainted with their teammates' expertise and social characteristics (Mortensen, 2014) and to learn the tacit aspects of their multiple, specific team roles (e.g., the assumptions and values present in a specific team; Biddle, 1986). Taken together, we hypothesize:

Hypothesis 2. An employee's multiple team membership is positively related to his or her hindrance appraisals.

\section{The Moderating Role of Organizational Tenure}

Importantly, we expect that the extent to which an employee perceives MTM as a challenge or a hindrance is contingent on his or her organizational tenure. An employee's 
organizational tenure reflects the total time employed at a specific organization and closely reflects his or her work experience within the organization (Ng \& Feldman, 2011).

Organizational socialization theory (Louis, 1980) suggests that increased organizational tenure goes along with greater interpersonal familiarity with clients, co-workers, and direct supervisors related to one's position in the organization, as well as improved understanding of the organization's work processes and implicit norms and values (Bauer et al., 2007; Gregersen, 1993). We propose that these socialization experiences may shape how employees appraise the need to work on multiple and varied tasks within the organization and to participate in complex collaborative work arrangements.

Scholars have argued that less experienced employees (i.e., with lower organizational tenure) prefer relatively stable task environments and smaller interpersonal settings (Louis, 1980; Jones, 1986). Employees with higher organizational tenure, by contrast, can wield their superior experience to better cope with complex work roles that require additional skills and larger social networks within the organization (Bauer et al., 2007; Gregersen, 1993). Consequently, they may be more able to grasp the requirements associated with MTM, as compared with lower-tenure employees, and may therefore perceive MTM as a demanding but manageable work practice (Biddle, 1986). In fact, MTM may enable experienced employees to improve their managerial and interpersonal skills and their standing within the organization, by working with a greater variety of people, managing overlapping deadlines, and resolving intra- and inter-team conflicts (Pluut et al., 2014). As a result, increasing MTM may strongly associate with positive challenge appraisals among these employees. We therefore hypothesize:

Hypothesis 3. Organizational tenure moderates the positive relationship between an employee's multiple team membership and his or her challenge appraisals, so that this relationship is stronger for employees with higher (rather than lower) tenure.

Organizational tenure may also moderate the relationship between MTM and hindrance appraisal. Employees with lower organizational tenure, on the one hand, need to direct substantial time and energy towards learning the interpersonal requirements and implicit norms of each of their teams within the organization (Ashforth \& Saks, 1996; Chao et al., 1994). Hence, higher MTM may render it more difficult for these employees to 'learn the ropes' of each respective team role, as employees are left with little time and resources to establish clear expectations within each specific team. We therefore expect that low-tenure employees are particularly likely to perceive high MTM as a type of hindrance. Employees with higher organizational tenure, on the other hand, may be relatively familiar with the people and parties involved in different organizational teams (Louis, 1980). Consequently, employees with higher tenure may retain greater perceptions of role clarity and an overall understanding of their position within the organization, even with high MTM, such that they are less likely to perceive MTM as a negative hindrance.

Hypothesis 4. Organizational tenure moderates the positive relationship between an employee's multiple team membership and his or her hindrance appraisals, so that this relationship is weaker for employees with higher (rather than lower) tenure.

\section{MTM, Job Performance, and Absenteeism: Conditional Indirect Relationships}


Taken together, our theorizing suggests that MTM is positively related to challenge appraisals, with organizational tenure strengthening this association. Extending our argumentation, we further draw from research on the challenge-hindrance framework to cast challenge appraisal as a predictor of job performance (Lepine \& Lepine, 2005). Hence, we propose that MTM is indirectly and positively related with an employee's job performance, with organizational tenure shaping the strength of this association.

Hypothesis 5. An employee's MTM is positively and indirectly related to his or her job performance, through challenge appraisals. This indirect relationship is more pronounced among employees with higher (rather than lower) organizational tenure.

Moreover, it is well established that hindrance appraisal is a key source of lowered job performance and absenteeism (LePine, Podsakoff, \& LePine, 2005; Moreau et al., 2004). Taken together, our argumentation suggests that MTM is positively related to hindrance appraisals, with organizational tenure strengthening this association. Hindrance appraisals, in turn, may associate negatively with job performance and positively with absenteeism. Hence, we hypothesize:

Hypothesis 6. An employee's MTM is negatively and indirectly related to his or her job performance, through hindrance appraisals. This indirect relationship is more pronounced among employees with lower (rather than higher) organizational tenure.

Hypothesis 7. An employee's MTM is positively and indirectly related to his or her absenteeism, through hindrance appraisals. This indirect relationship is more pronounced among employees with lower (rather than higher) organizational tenure.

\section{METHODS}

\section{Data and Study Design}

We collected multi-source archival data from a large organization in the Netherlands that conducts applied contract research in a wide variety of knowledge areas for both private and public institutions. The organization provided weekly work hour data, along with information on individual absenteeism and job performance, for all research employees for two consecutive years. Additionally, we drew on survey data, as available from the host organization's bi-annual employee satisfaction survey, to assess employees' challenge and hindrance appraisals. We received data for all 1706 researchers employed at the organization during the study period. After matching data from different sources, we retained a usable sample of $1211 \mathrm{employees}$ (effective response rate $=71 \%$ ).

Following the assumed causal sequence underlying our theoretical predictions, we used MTM at an earlier time point to predict outcomes at a later point in time (Arjas, \& Parner, 2004; Shin \& Konrad, 2014). Our measure for multiple team membership was based on work hour registrations from the first three quarters of 2010, whereas challenge and hindrance appraisals were operationalized with items from an employee satisfaction survey conducted in the final quarter of 2010. Absenteeism and job performance, in turn, pertained to the year 2011.

\section{Measures}


Multiple team membership. We measured MTM using employees' work hour registrations to capture the number of teams in which an employee was actively involved during any given week. We averaged these weekly measures across the study period.

Organizational tenure. We used the number of years an employee had worked at the organization to operationalize organizational tenure (Ng \& Feldman, 2011).

Challenge appraisals. Three items from the organization's employee satisfaction survey closely resembled our theoretical conceptualization of challenge appraisals. Specifically, employees were asked to rate, on a scale from 1 (low) to 10 (high), how satisfied they were with 'the amount of challenge in my work'; 'the extent to which my work is useful' and 'the extent to which I can use my creativity in my work' (Cronbach's alpha $=.81$ ).

Hindrance appraisals. To capture hindrance appraisals, we used three additional items from the organization's employee satisfaction survey. Employees were asked to rate, on a scale from 1 (low) to 10 (high), how satisfied they were with 'the extent to which I know what is expected of me'; 'the extent to which I have a clear understanding of what is going on in the organization' and 'the extent to which it is clear how my performance is evaluated' (Cronbach's alpha $=.72$ ). We reverse-coded all items.

Job performance. At the beginning of each year, the host organization's human resource management system required department-level supervisors to assess their direct reports' performance during the past year. A standardized evaluation was used to assess each employee's overall performance on a five-point scale, with 1 representing the worst possible evaluation (i.e., substantial need for improvement) and 5 indicating the best possible evaluation (i.e., highly effective and well-functioning).

Absenteeism. To operationalize absenteeism, we used the total number of sick leave hours in the year 2011 .

Control variables. We controlled for an individual employee's general workload (fulltime equivalents) and gender.

\section{RESULTS}

We used structural equation modeling in Mplus version 7.31 (Muthén \& Muthén, 19982011) to examine our hypotheses. Specifically, we used Ordinary Least Squares estimation procedures to examine Hypotheses 1-6, and a Zero Inflated Negative Binomial estimate to examine Hypothesis 7. The conditional indirect effects were assessed at both higher $(+1 \mathrm{SD})$ and lower (-1SD) levels of organizational tenure, using 10'000 bootstrap samples to estimate associated $95 \%$ confidence intervals (cf. Hayes, 2009).

We found no support for the positive association between MTM and challenge appraisal suggested in Hypothesis $1(B=-.02, p>.05)$. In support of Hypothesis 2 , however, MTM was positively related with employees' hindrance appraisals $(B=.09, p<.01)$. 
The interaction term for MTM and organizational tenure was positively associated with challenge appraisals $(B=.10 ; p<.01)$, although the respective interaction pattern did not match our prediction in Hypothesis 3. Specifically, the MTM-challenge appraisal linkage was nonsignificant (rather than positive) among employees with relatively high tenure (simple slope at $+1 \mathrm{SD}=.08, p>.05$ ), whereas this linkage was negative for employees with lower tenure (simple slope at $-1 \mathrm{SD}=-.12, p<.05)$. Hence, Hypothesis 3 was not supported. Corroborating Hypothesis 4, however, the interaction term for MTM and organizational tenure was positively related with hindrance appraisals $(B=-.10 ; p<.05)$. As suggested, the MTM-hindrance appraisal linkage was negative among employees with relatively low tenure (simple slope at $-1 \mathrm{SD}=.19, p<.05$ ), whereas this linkage was not significant for employees with higher tenure (simple slope at $+1 \mathrm{SD}$ $=.00, p>.05$ ).

Regarding Hypothesis 5, the indirect relationship between MTM and job performance, through challenge appraisals, was non-significant among employees with relatively high tenure (estimate at $+1 \mathrm{SD}=.01, p>.05)$, whereas MTM was indirectly and negatively related with job performance (through reduced challenge appraisal) when tenure was lower (estimate at $-1 \mathrm{SD}=$ $.01, p<.05)$. Hence, Hypothesis 5 was not supported. That is, we hypothesized that MTM indirectly improved performance when tenure was high, while in fact, MTM lowered job performance (mediated by lower challenge appraisals) when tenure was low.

Further, supporting Hypothesis 6, we found a negative indirect relationship between MTM and job performance, via hindrance appraisal, when organizational tenure was lower (estimate at $-1 \mathrm{SD}=-.03, p<.05$ ), whereas this indirect linkage was not significant with higher tenure. Finally, as predicted in Hypothesis 7, we found a significant and positive indirect relationship between MTM and absenteeism (through hindrance appraisal) among employees with lower organizational tenure (estimate at $-1 \mathrm{SD}=.10 ; p<.05$ ), but not among employees with higher tenure (estimate at $+1 \mathrm{SD}=.00 ; p>.05$ ).

\section{DISCUSSION}

Our findings demonstrate that some employees are more susceptible to MTM's consequences than others. Multi-teamers with relatively low organizational tenure experienced a decrease in challenge appraisal, and, subsequently lower job performance. Furthermore, these employees appraised MTM as a clear-cut hindrance, subsequently decreasing their performance and increasing absenteeism. For multi-teamers with higher organizational tenure, by contrast, MTM was unrelated with both challenge and hindrance appraisals and, accordingly, it did not decisively shape their job performance or absenteeism. As such, it appears that research needs to move beyond claims that MTM has either positive or negative implications, instead focusing on key moderating variables that may shape employees' reactions to such complex work arrangements.

We acknowledge that the present study has a number of limitations that future research may address, including our use of archival data sources (rather than more established instruments; cf. Pearsall, Ellis, \& Stein, 2009) to capture challenge and hindrance appraisals. Nevertheless, organizations seem well advised to limit new employees' concurrent membership in multiple teams and to only gradually increase employees' MTM as they gain more experiences within the organization.

\section{REFERENCES AVAILABLE FROM THE AUTHORS}

